\title{
Automatic Detection of Flood Using Remote Sensing Images
}

\author{
Amith Chandrakant Chawan, \\ Electronics and communication Department. \\ MMEC, VTU, Belagavi \\ Email: camitkumarchavan@gmail.com \\ Vaibhav K Kakade, \\ Electronics and communication Department. \\ MMEC, VTU, Belagavi \\ Email: kakade.ece@gmail.com

\section{Jagannath K Jadhav} \\ Electronics and communication Department. \\ MMEC, VTU, Belagavi \\ E-mail: jagannathjadhav3030@ gmail.com
}

\begin{abstract}
Remote sensing imaging (RSI) technology has recently been identified as an effective photogrammetric data acquisition platform to rapidly provide high resolution images due to its profitability, its ability to fly at low altitude and the ability to analysis in dangerous areas. The various kinds of classification techniques are have been used for flood extent mapping for finding the flood affected region, but based on the color region based analysis the classified hazardous area has very complex. Due to over the above issues in this work there significant enhancements have appeared in the classification of remote sensing images using Contiguous Deep Convolutional Neural Network (CDCNN).In the flood detection system the four different kinds of process like preprocessing, segmentation, feature extraction and the Contiguous Deep Convolutional Neural Network (CDCNN) has been executed for identifying the flood defected region. This works also investigates and compare with the possible methods with the proposed CDCNN for accurately identified by the Classification details of the RSI
\end{abstract}

Keywords: Contiguous Deep Convolutional Neural Network, Flood Detection, Remote Sensing Images.

\section{Introduction}

Remote sensing has huge improvement in the modern era for identifying and categorizing objects present on earth [1,17]. Catastrophic events, such as floods, landslides and tsunamis, have a major impact on living being lives because of this disaster causes a significant loss of life and property[2,3]. Flood disasters are the most frequent natural misadventures in the worldwide and can become an important area of concern for the consequences of future climate change [4,5,6]. This kind of consequences, are mainly supervised including flood monitoring, to be an important issue for the country. Flood monitoring requires quick access to important information about the extent of flooding and changes in land cover. Using remote sensing data, they are techniques for detecting information 
change on objects or phenomena obtained without direct physical contact, and all can be used to estimate information in rapid time frames for flood monitoring [7,8,9]. From the analysis of detection process can identifying various kind of state of an object or phenomenon by observing at different times. In mean Time an accurate detection of flood change provides a better understanding of the disaster situation and helps establish the basis for disaster recovery plans $[10,11,12]$. The availability and quality of multi-temporal remote sensing data has been developed, and the progress of change detection technology has increased for flood monitoring using multi-temporal satellite images in recent years with a growing interest in monitoring.

This work is an outcome of the project "Flood and damage assessment using very Multi-Temporal Remote Sensing Images (MT-RSI) data". Remote sensing technology has played an essential role in flood monitoring in recent years. This development (optical/aerial to radar remote sensing) offers all weather capability as associated with the optical sensors for the purpose of flood mapping. Flood mapping is a technique used for flood monitoring, in which the front and rear flood images are compared to classify an updated (not flood) and flood area. Initially, flood monitoring was limited to satellite and aerial images. But using Remote sensing image classification is widely used for terrestrial data classification. The Classification model based on first-order statistics, texture measures based on the classification model, cluster-based classification model, convolutional neural network model, mainly component-based classification model and multisensory fusion based on (various resolution based image classification) is a general classification model that uses different software programs.

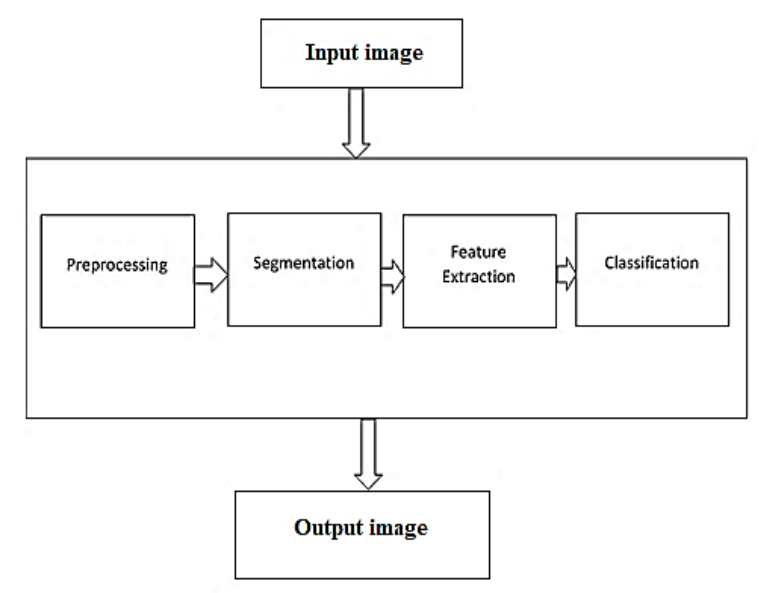

Figure.1 Functional block diagram for the Remote Sensing Image Processing

\section{LITERATURE SURVEY}

There is different kind of techniques used for image enhancement, segmentation, feature extraction and classification are used for Flood detection system which are described in the literature. Also discussing existing techniques and its limitations. The complexity of remote sensing data processing is explored; there is a crucial need for a better classifier is absorbed for the proposed ideology. 
Ohki, M., (2019) The analysis of Flood disasters in the Kanto and Tohoku regions, Japan's investigation of flooded areas that obtained PALSAR-2 interference data during the detection of urbanized areas. The results show that threshold flood detection through phase standard deviation (PSD) images is more accurate than consistent. Our continuous work is to use more new technologies to further improve the accuracy of interferometric phase details. Another imminent issue is the misidentification of disasters not related to seasonal and time changes. It requires more data, and the study currently uses only a time series analysis of a pair of interference data.

Chenwei Deng, (2019) Cloud detection is an essential task for RS image processing. Several cloud detection techniques has been created. However, most conventional system eliminate the effects of the thin cloud layer and do not have the ability to discern areas where clouds are metrically similar, such as buildings and snow. This system utilizes anew cloud detection techniques for RSI, the test images are classified into three categories: thick clouds, thin and non-cloudy clouds. First, a simple linear iterative grouping algorithm uses the potential to segment clouds, including small clouds. Then, a statistical system of aRSI is applied to distinguish super-pixel clouds from surface buildings.Finally, Gabor's characteristics are calculated on each super pixel and a support vector machine is used to distinguish clouds from snowy regions. The simulation outcomes show that the implemented model is superior to cutting-edge methods for cloud detection

Young-Joo Kwak,(2018) For improving the exact region based flood mapping the Support vector machine (SVM) is implemented, Integration of multiple satellite data sources is essential Maximum capacity and compensation for sensor with the filters limitations for Optical data and SAR. The main objective of SAR research is Use of algorithms that Flood mapping and synthetic aperture radar (SAR) images Compare and combine two different statistical thresholds Focus to enhance flood detection regions, images Survey fusion technology is maximizing Calibration and optimization of floods using mapping as Integral approach to flood detection. To show operational characteristics of MODIS Landsat-8 data Acquisition.

Marais, W. (2017) this method considers the de-noising and reconstructing the corrupt noise images. Poisson noise ascends in the circumstance of counting or scattering of photons. In the different use domains of astronomy and medical imaging, the number of photons is very low, resulting in very low signal-to-noise ratio images. This system an Azzari is investigated using a 3D for Poisson image denoising in a high resolution framework. Especially, the de-noised result at a coarse resolution is utilized to increase the de-noising of the next better resolution, resulting in state-of-the-art de-noising outcome. This method provides the correct alternative normalized maximum likelihood formulation for the modernization issues and illustrates that it can be resolved by using the gradient optimization algorithm near the coarse first well. The methods proposed in this paper are compared for Implemented methods and their solid similarities.

Network-based computing, a distributed data processing system in which each computer node is connected to other independent computers through a network, is discussed by Eremenko, (2018). The 
method of speed calculation is presented in the form of a so-called "scheme" of the system. The "solution" is a formal description of a solution to a task within the framework of a distributed processing system. When the plan starts, it is used to control the serial number during the execution of the task. All connections between the solution server and the processor are made with the Secure Shell (SSH) protocol.

\section{Proposed MeTHOdOLOGY}

The methodology mainly divided in to three stages which are as follows

- $\quad$ Preprocessing

- $\quad$ Multi model clustering segmentation technique

- $\quad$ Feature Extraction

- Contiguous Deep Convolutional Neural Network (CDCNN) classification

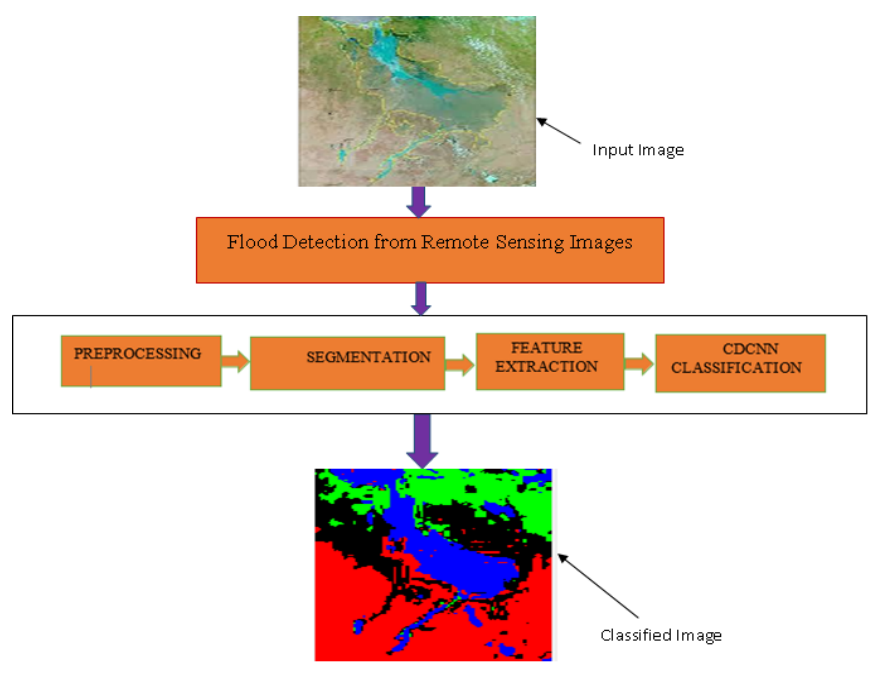

Figure.2 Proposed system Block diagram 


\subsection{Pre-processing}

The method is essential process for most image analysis tasks aiming at decomposing an image into regions having visual similarity. It is a process to seek out for normal regions in an image and preprocessing these noise regions. This is achieved because the first two are not substantial enough to completely eliminate the possibility of multiple responses using Gaussian filter. Second Mainly preprocessing technique considers Image Enhancement that provides an accurate color variation in the image processing. Mainly the histogram analysis of a normal image refers as the histogram of pixel intensity and its values. It then finds the image gradient to highlight regions with high efficient image response.

Preprocessing Steps are as follows

Step1:Start

Step2:Read the input-Satellite image Data, Image height, Image width, Training data.

Step3:The filter mask of the enhanced mask is primarily a square mask or a mask cross. Considering the symmetry of the mask, $n$ is often odd ranges.

Step4:Construct an image. The array of 256 values that comprises the number of pixels with the value $\mathrm{x}$

Step5:Calculate the density function ( $\mathrm{f}[\mathrm{x}])$. This is a 256 value array containing the number of pixels with value.

Step6:for every pixel i (with grey level) in the image do.

Step7:compute objective function

Step8:Based on the average and improved algorithms, impulse noise filtering algorithms with a density of $10 \%, 35 \%$ and $45 \%$ were added to the original image analysis

Step 9: Stop 


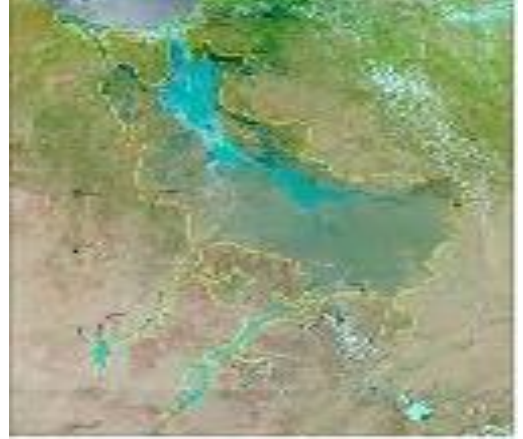

Figure. 3(a) input Filter

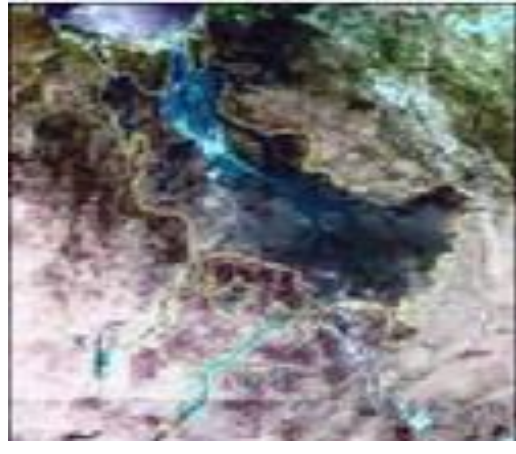

Figure. 4(b) Preprocessing Image

\subsection{Multi model clustering segmentation technique.}

These techniques are used in images related to objects for pixel segmentation. Threshold technology is linked to region-based segmentation, which can easily detect flooded areas by recognizing color images. This is the area where segmentation detection should be disabled. Region-based segmentation is also called" Similarity Based Segmentation" On the basis of this, three criteria of Multi model clustering are proposed. Multi model clustering uses maxima of the gradient image to find clear view.

Image clustering detection is more common in gray levels than remote image detection and fine line detection discontinuities because isolated points and fine lines do not occur frequently in most real images. The boundary between the two regions formed by the group has a characteristic of a relatively different level. Here it is assumed that the transition between the two regions can be attributed. Also that the transition between two or more regions can be determined on the basis of individual preprocessing level discontinuities. The amount of noise should also be measured, because noise can ignore a part of the form or a part added to it. For region based multi-temporal images.

- Changing the information image into a feature portrayal

- Observe the first image information to get the portrayal in six color channels

- Recognizing the cluster focuses and number

- Calculating the thickness $\rho$ and remove $\delta$ by utilizing the condition. And afterward creating the choice chart given the thickness and separation

- Before presenting according to the rules, select the data points with high density $(\rho)$ and a considerable distance $(\delta)$ as the center of the clusters. Then can calculate the cluster number

- Assign the continuing points to the cluster.

- If the information point is nearest to its cluster, abandon it where it is. On the off chance that the information point isn't closest to its cluster, move it into the nearest cluster.

- Accomplish final segmentation based on labeling until the last step, as shown in Figure 4b. 


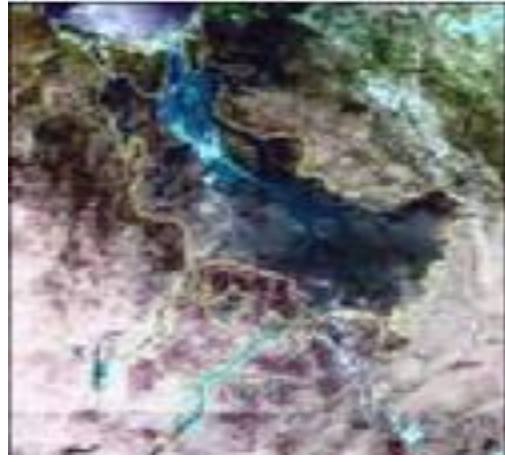

Figure. 4(a) input Filter

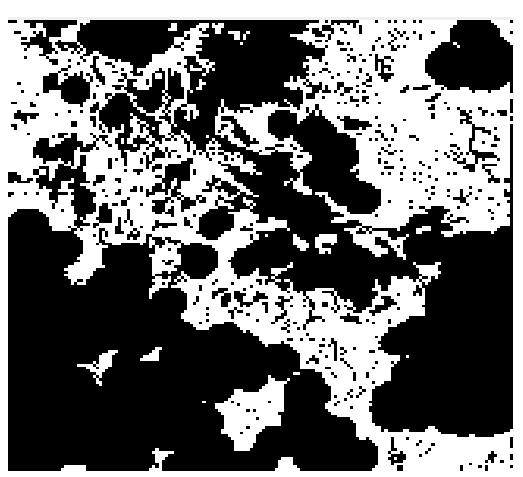

Figure. 4(b) segmentation Image

\subsection{Feature Extraction.}

Automatic extraction of a feature from great determination remotely detected imagery isn't just deductively difficult yet also of major pragmatic significance for information procurement and refresh of Geographic Information System (GIS) databases. The second section of the classification module sis feature extraction. Subsequently this segment delivers a huge effect on the consequences of classification, it assumes an essential part in the implementation of any image classification. Feature extraction is a kind of dimensionality decrease that productively speaks to intriguing parts of an image as a minimized feature vector. In this Texture Vector Zone method is projected to extract the features RSI. The algorithm steps of Feature extraction is discussed below.

Table 1 Features Extracted For RSI

\begin{tabular}{|l|l|}
\hline \multicolumn{1}{|c|}{ Feature Category } & Feature Details \\
\hline Geometric Features & Boundary,regions \\
\hline Texture features & Energy, Entropy,Homogeneity, Varience \\
\hline Color features & Mean, Standard Deviation, Skew, Kurtosis \\
\hline Fractal features & Standard Deviation Fractal Dimension \\
\hline
\end{tabular}

Mean:

The calculation of the average signal filter is a variation of the arithmetic average filter and is mainly used for images with Gaussian noise.

$$
f(x, y)=[\eta] g(r, c)^{1 / \min }
$$


The arithmetic mean is the average of the values $\{\mathrm{x} 1, \mathrm{x} 2 \ldots . . \mathrm{xn}\}$ located within a time window.

Standard deviation:

This is the most commonly used metric for degeneration or decent variety used as part of the information. As for the preparation of images, it shows how many varieties or "dispersions" exist from normal values (average or expected).

$$
S D=\sqrt{\frac{\Sigma|x-\mu|^{2}}{N}}
$$

Where $\Sigma$ means "sum of the function", $\mathrm{x}$ is a value in the images, is the mean of the image and $\mathrm{N}$ is the number of data points in the image

Variance:

The variance is computed how the numbers are arranged. This is one of several descriptors for a probability cycle, which represents how far the average number is (expected self-esteem). Specifically, the difference is one of the snapshots of a grant.

$$
\sigma^{2}=\frac{1}{N} \sum_{i=1}^{N}\left(x_{i-}\right.
$$

Where, is the image vector and $\bar{x}$ is the mean given by: $1 / \mathrm{N}$ 
Entropy:

$\mathrm{H}$ is the entropy of the random variable. The, entropy can be used to classify textures, can have a certain entropy, and certain patterns are repeated in definite methods.

$$
\mathrm{H}=-\sum_{\mathrm{i}=1}^{\mathrm{n}} \mathrm{p}_{i} \log _{2}\left(\mathrm{p}_{i}\right)
$$

\subsection{Contiguous Deep Convolutional Neural Network (CDCNN) classification}

The project uses the CDCNN architecture in the literature, but its basic constituents are very similar. The characters recognized by a typical convolutional network architecture. CDCNN are multilevel trainable architectures and each stage consists of multiple layers. The entry and exit of each stage is a set of matrices called feature maps. In the case of color images, each feature map will be a color channel that contains the input image, a 3D matrix for video and a one-dimensional matrix, and a 2D matrix for audio input. This exit stage represents the characteristics extracted from all the entered positions. Each stage generally consists of a convolutional layer, a nonlinear layer and a group layer. There are single or multiple layers completely connected after several convolutional and grouping layers.

1). CDCNN technique

Step 1:Select the Input image.

Step 2:Apply Gaussian filter for preprocessing

Step 3:Apply multi model clustering segmentation method for preprocessing image segmentation

Step 4:To extract the features from segmentation's image using Feature extraction method.

Step 5:Get the comparable feature elements

Step 6:Apply the Contiguous Deep Convolutional Neural Network (CDCNN) Classification method to train the model. 
DOI: https://doi.org/10.36548/jitdw.2020.1.002

a) Fix the threshold Value in every pixels

b) Discover the Structural analysis for every pixel.

Step7:Check the threshold range with Discovered Structural analysis.

a) Arrange every pixel to interface the parts and to consolidate the districts.

Step8: Go to step 6

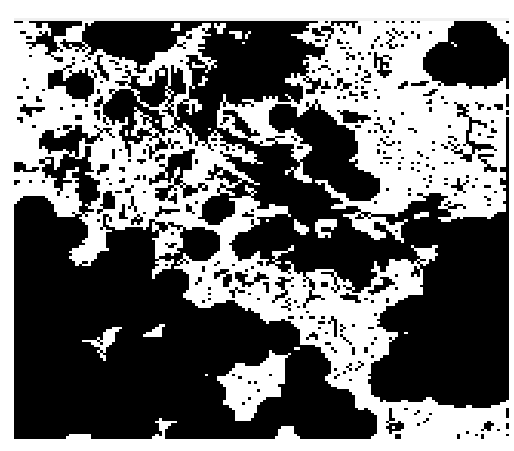

Figure.5(a) Segmentation Image

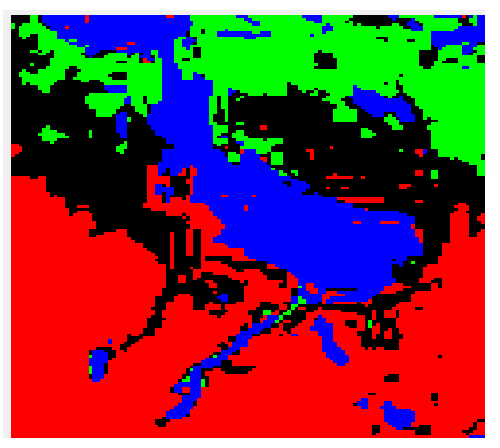

Figure.5(b) Classification Image

2). Classification features

Precision: This is the proportion of a class of positive examples that are exactly organized in the total number of tests.

True Positive State(TPS)

True Positive State(TPS) + False Positive State (FPS)

Recall: This is a precise grouping to test some positive weights instead of positive collective numbers.

$$
\text { Recall }=\frac{\text { True Positive State }(\text { TPS })}{\text { True Positive State(TPS) }+ \text { False neagtive State (FNS) }}
$$


F-measure: It is the particular harmonic precision and recall given by the following formula.

$$
F_{\text {measure }}=\frac{2 * \text { Precision } * \text { recall }}{\text { precision }+ \text { recall }}
$$

3). flow chart.

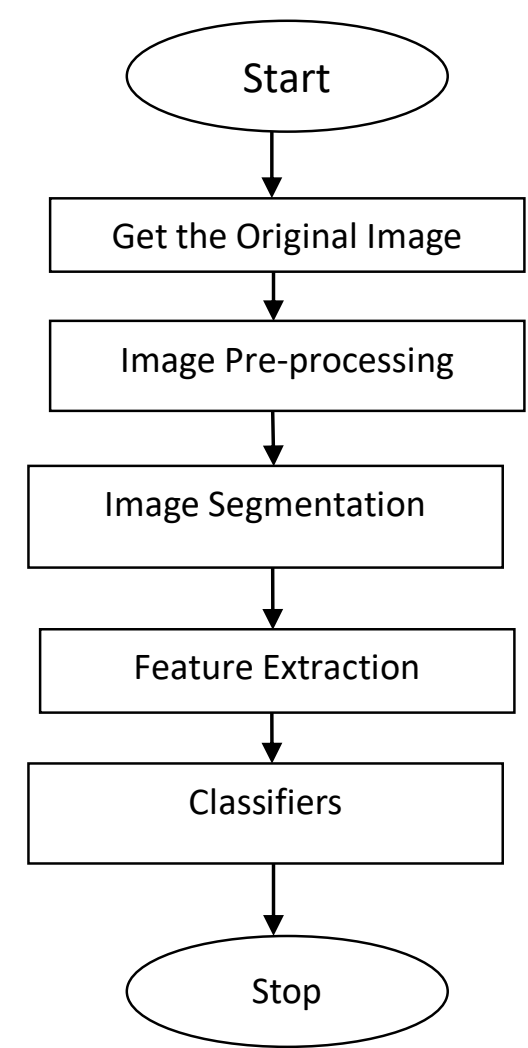

Figure.7 Flow Chart

\section{EXPERIMENTAL RESULTS}

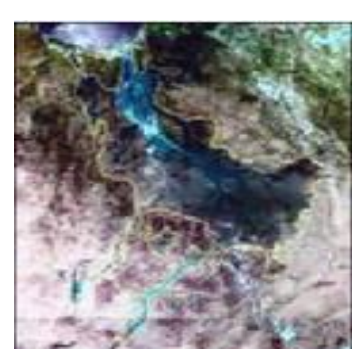

Figure.6 (a) input

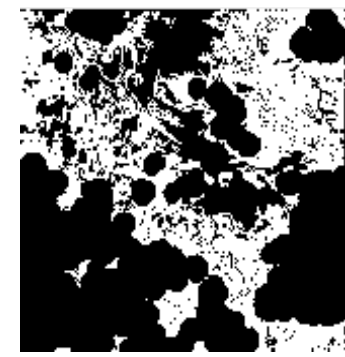

(b) Segmentation Image

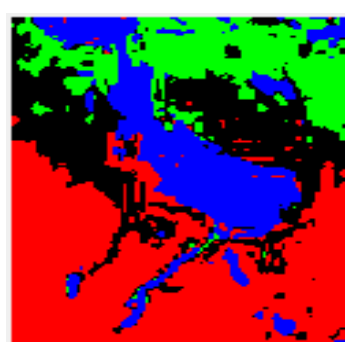

(C) Classification 
Figure $6 \mathrm{a}, \mathrm{b}$ and $\mathrm{c}$ it is shown that the same steps for the second data set are repeated for the second entry and the increase in grouping results in greater precision in the number of segmentation results for the grouping segmentation processing steps. Since Table 1 shows the evaluation parameters obtained using the proposed technique. Clustering systems are being used to order information and perceptions in a couple of portions, so information inside any area are relating, while information crosswise overlayers is extraordinary. The Image upgrade is the way toward altering advanced images with the goal that the outcomes are more reasonable for show or further image investigation.

Table 2 Represents the performance Analysis for Remote sensing images, in this analysis five different types of images are verified with its considering some features like mean, standard deviation, variance, MS and Entropy for the classification result.

Table: 2 Feature Extraction Result for Data Set-1

\begin{tabular}{|l|l|l|l|l|l|}
\hline $\begin{array}{l}\text { Sample } \\
\text { no }\end{array}$ & $\begin{array}{l}\text { Mean } \\
(\mathbf{d B})\end{array}$ & $\begin{array}{l}\text { Standard } \\
\text { deviation } \\
(\mathbf{d B})\end{array}$ & $\begin{array}{l}\text { Variance } \\
(\mathbf{d B})\end{array}$ & $\begin{array}{l}\text { RMS } \\
(\mathbf{d B})\end{array}$ & $\begin{array}{l}\text { Entropy } \\
\text { (joule) }\end{array}$ \\
\hline DSS- 1 & 0.00871 & 0.0597566 & 0.03564 & 0.059761 & 3.33755 \\
\hline DSS-8 & 0.00652 & 0.0569842 & 0.09435 & 0.089565 & 4.56953 \\
\hline DSS- 3 & 0.00762 & 0.0679356 & 0.06789 & 0.047896 & 5.03215 \\
\hline DSS- 6 & 0.00573 & 0.0835651 & 0.04356 & 0.061239 & 2.01398 \\
\hline DSS-3 & 0.04179 & 0.0798656 & 0.02365 & 0.010569 & 6.09872 \\
\hline
\end{tabular}




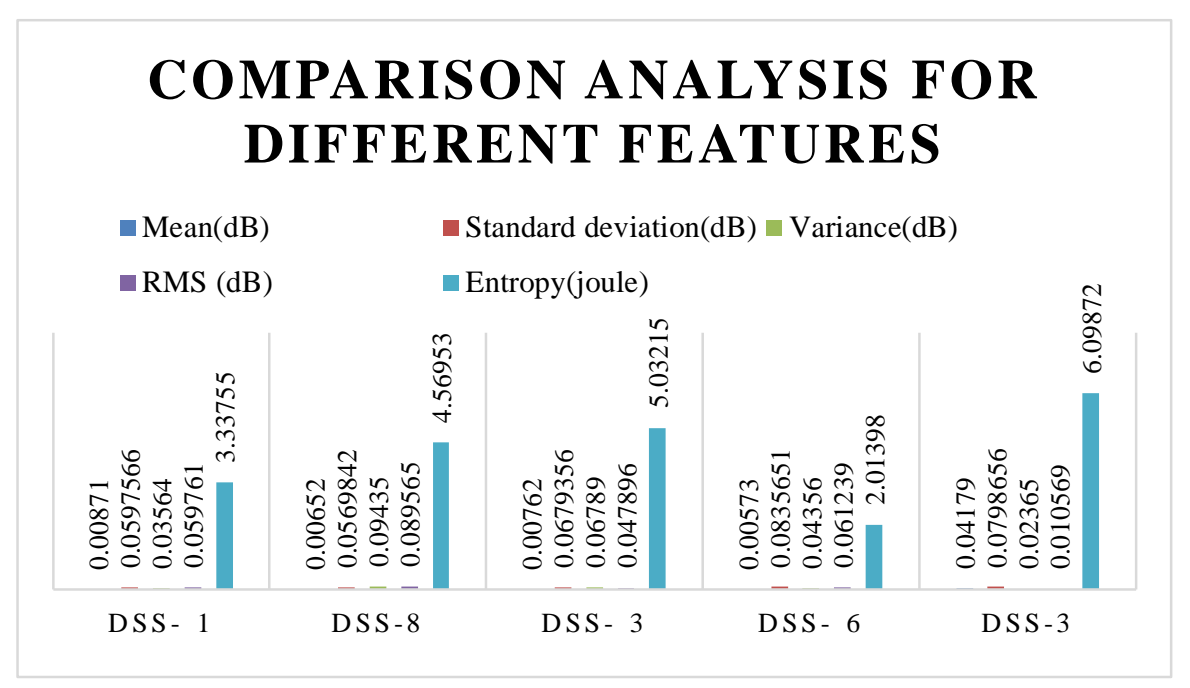

Figure.7 Comparative analysis

Figure 7 shows that the comparative analysis for the feature extraction in the Remote sensing image processing mainly considered some features like mean, standard deviation, variance, and Entropy and RMS values. The values of all the extraction are prescribed in table -2

TABLE 3: DIFFERENT CLASSIFICATION FEATURES FOR TWO DATASET HIERARCHY

\begin{tabular}{|l|l|l|l|}
\hline Methods & Precision & Recall & F-measure \\
\hline Convolutional Neural Network & 0.893214 & 0.645953 & 0.793656 \\
\hline $\begin{array}{l}\text { Contiguous Deep } \\
\text { Convolutional Neural Network } \\
\text { (CDCNN). }\end{array}$ & 0.916479 & 0.8960094 & 0.877626 \\
\hline
\end{tabular}

Table 3 shows the different classification features by evaluating the flood region using CDCNN. The proposed technique precision is high as conventional technique. The results clarify that the important point here is the processing time, which is recorded to include several RSI images because it is less complicated than the others. 


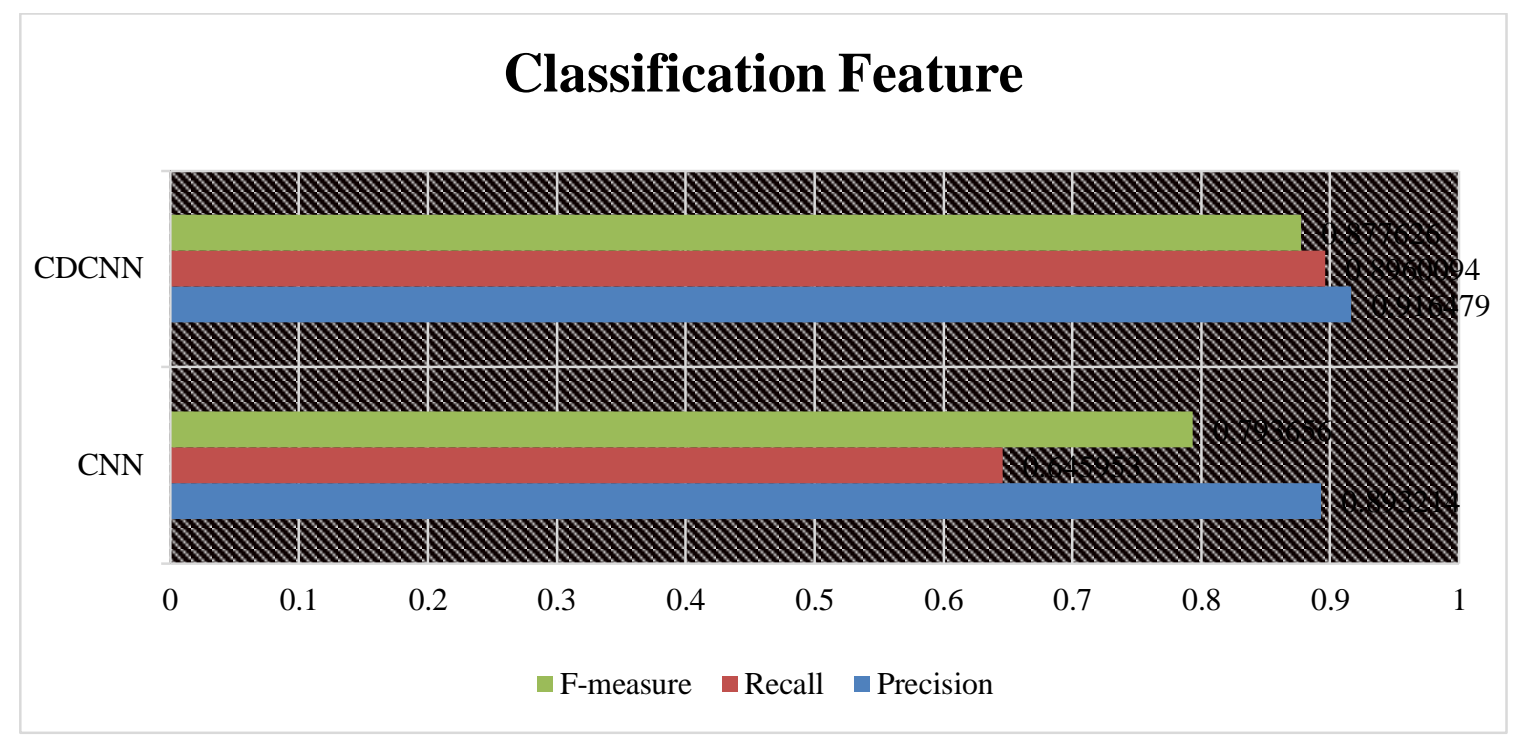

Figure 8 Evaluation classification features

The figure 8 specified the Evaluation classification features quantities precision; recall got for the proposed and existing technique.

\section{CONCLUSIONS}

This paper developed the implementation of work Contiguous Deep Convolutional Neural Network $(\mathrm{CDCNN})$ classification is used for recognize the flood region using remote sensing image. The proposed Remote Sensing Images classifier has produced accurate flood detect with less false classification ratios $2 \%$. The performance of the proposed system has been confirmed by simulation using MATLAB software.

\section{References}

[1] Jagannath K Jadhav, Amruta P Sonavale, R.P.Singh,( 2019).Segmentation Analysis Using Particle Swarm Optimization -Self Organizing Map Algorithm and Classification of RemoteSensing Data for Agriculture. Lecture Notes on Data Engineering and Communications Technologies Volume 38, p.659-668.

[2] Ohki, M., \& Shimada, M. (2019). Flood Detection in Built-up Area Using Interferometric SAR Data by Palsar-2. IGARSS 2019 - 2019 IEEE International Geoscience and Remote Sensing Symposium, Conference Location: Yokohama, Japan.

[3] Sui, H., An, K., Xu, C., Liu, J., \& Feng, W. (2018). Flood Detection in PolSAR Images Based on Level Set Method Considering Prior Geo-information. IEEE Geoscience and Remote Sensing Letters, Volume: 15, No.5, PP. 699 - 703.

[4] V. Klemas,( 2014). "Remote Sensing of Floods and Flood-Prone Areas: An Overview," J. Coast. Res., pp. 1005-1013.

[5] N. M. Robertson and T. Chan, "Aerial image segmentation for flood risk analysis," in 2009 16th IEEE International Conference on Image Processing (ICIP), 2009, pp. 597-600. 
[6] S.-W. Lo, J.-H. Wu, F.-P. Lin, and C.-H. Hsu, "Visual Sensing for Urban Flood Monitoring," Sensors, vol. 15, no. 8, pp. 20006-20029, 2015.

[7] M. Geetha, M. Manoj, A. S. Sarika, M. Mohan, and S. N. Rao, "Detection and estimation of the extent of flood from crowd sourced images," in 2017 International Conference on Communication and Signal Processing (ICCSP), 2017, pp. 0603-0608.

[8] Ziming Miao, Kun Fu "Automatic water-body segmentation from high-resolution satellite images via deep networks" in IEEE geoscience and remote sensing letters, Vol. 15, No. 4, 2018.

[9] Villa, Alberto \&Benediktsson, (2012). 'Hyperspectral Image Classification with Independent Component Discriminant Analysis'. Geoscience and Remote Sensing, IEEE Transactions on. 49. 4865 - 4876. 10.1109/TGRS.2011.2153861.

[10] B. Pan, Z. Shi, and X. Xu, "MugNet: Deep learning for hyper spectral image classification using limited samples,' ISPRS J. Photogramm. Remote Sens., vol. 145, pp. 108-119, Nov. 2018.

[11] W. Li and Q. Du, "Joint within-class collaborative representation for hyperspectral image classification," IEEE J. Sel. Topics Appl. Earth Observer. Remote Sens., vol. 7, no. 6, pp. 2200-2208, Jun. 2014.

[12] Zhongleren, biaohou, (2018) "Patch-sorted deep feature learning for high resolution SAR image classification" in IEEE journal of selected topics in applied earth observations and remote sensing, Vol. 11, No. 9.

[13] Ohki, M., \& Shimada, M. (2019). Flood Detection in Built-up Area Using Interferometric SAR Data by Palsar-2. IGARSS 2019 - 2019 IEEE International Geoscience and Remote Sensing Symposium,Conference Location: Yokohama, Japan.

[14] Chenwei Deng, Zhen Li, "Cloud Detection in Satellite Images Based on Natural Scene Statistics and Gabor Features" in IEEE Geoscience and remote sensing, Vol. 30, No. 3, pp. $485-491,2019$.

[15] Young-JooKwak, Ramona Pelich, Jonggeol Park, Wataru Takeuchi (2018). Improved Flood Mapping Based On The Fusion Of Multiple Satellite Data Sources And In-Situ Data. IGARSS 2018 - 2018 IEEE International Geoscience and Remote Sensing Symposium,Conference Location: Valencia, Spain.

[16] Marais, W., \& Willett, R. (2017)"Proximal-Gradient methods for Poisson image reconstruction with BM3D-Based regularization", in International workshop on computational advance in multi-sensor adaptive processing.

\section{Authors Biography}

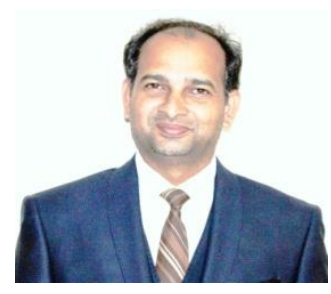

I have many years' experience as Assistant Professor in engineering colleges. Handled various subjects in Electronics \& Communication Engineering Department and has Strong synthesizing, writing, and information technology skills: wrote scientific articles in international peer-reviewed journals, as well as the highly technical 
Journal of Information Technology and Digital World (2020)

Vol.02/ No. 01

Pages: $11-26$

https://www.irojournals.com/itdw/

DOI: https://doi.org/10.36548/jitdw.2020.1.002

replies to the questions raised by the reviewers. Areas of Research Interest are Digital signal processing, DSP algorithms and architectures, Image Processing, Remote Sensing Applications, Signals and Systems. I have more than seven journals papers published in Scopus indexed journals. I have Applied Three patents which are published in Indian patent journals. 